\title{
The electronic structure and reflectivity of PEDOT:PSS from density functional theory
}

\author{
Annika Lenz, Hans Kariis, Anna Pohl, Petter Persson and Lars Ojamäe
}

\section{Linköping University Post Print}

N.B.: When citing this work, cite the original article.

Original Publication:

Annika Lenz, Hans Kariis, Anna Pohl, Petter Persson and Lars Ojamäe, The electronic structure and reflectivity of PEDOT:PSS from density functional theory, 2011, Chemical Physics, (384), 03-jan, 44-51.

http://dx.doi.org/10.1016/j.chemphys.2011.05.003

Copyright: Elsevier Science B.V., Amsterdam.

http://www.elsevier.com/

Postprint available at: Linköping University Electronic Press

http://urn.kb.se/resolve?urn=urn:nbn:se:liu:diva-69856 
The electronic structure and reflectivity of PEDOT:PSS from density functional theory

\author{
Annika Lenz ${ }^{1,2}$, Hans Kariis ${ }^{3}$, Anna Pohl $^{3}$, Petter Persson ${ }^{2,4}$, Lars Ojamäe ${ }^{1}$ \\ ${ }^{1}$ Physical Chemistry, IFM, Linköping University, SE-58183 Linköping, Sweden \\ ${ }^{2}$ Chemical Physics, Lund University, SE-22100 Lund, Sweden \\ ${ }^{3}$ Swedish Defence Research Agency, FOI, SE-58330 Linköping, Sweden \\ ${ }^{4}$ Theoretical Chemistry, Lund University, SE- 22100 Lund, Sweden
}

\begin{abstract}
The geometric and electronic structure of condensed phase organic conducting polymer PEDOT:PSS blends has been investigated by periodic density functional theory (DFT) calculations with a generalized-gradient approximation (GGA) functional, and a plane wave basis set. The influence of the degree of doping of the PEDOT polymer on structural and optical parameters such as the reflectivity, absorbance, conductivity, dielectric function, refractive index and the energy-loss function is studied. A flip from the benzoid to the quinoid structure is observed in the calculations when the neutral PEDOT is doped by negatively charged PSS. Also the optical properties are affected by the doping. In particular, the reflectivity was found to be very sensitive to the degree of doping, where higher doping implies higher reflectivity. The reflectivity is highly anisotropic, with the dominant contribution stemming from the direction parallel to the PEDOT polymer chain.
\end{abstract}




\section{Introduction}

PEDOT, poly(3,4-ethylenedioxythiophene) [1-4], is a conductive polymer [5] that can be used in many different applications such as antistatic coating of polymers and glass, high conductive coatings, organic LED-(OLED) displays [6], nano-fiber electrodes for cell stimulation [7], solar cells, cathode material in electrolytic capacitors, printing wiring boards [8], textile fibres with colour changing properties [9], transparent electrodes for thick-film electroluminescence [10], source gate and drain in the rapidly developing organic semi-conductors field [11].

In experimental applications PEDOT is stabilized by counter ions such as PSS, poly(styrenesulfonate), in PEDOT:PSS. It has been observed that the conductivity increases when certain organic solvents has been added to a PEDOT:PSS water solution [12]. Some examples are dimethyl sulfoxide (DMSO), N,Ndimethylformamide (DMF) [13], glycerol, sorbitol [14-15] and ethylene glycol [16]. The mechanism behind the change in conductivity in PEDOT:PSS when exposed to different solvents has been studied with several experimental methods. An increase in conductivity was observed only when the organic solvent contained two or more polar groups. These molecules interact with dipoles or charges on the PEDOT chains and cause a transformation from benzoid to quinoid structure [12]. This will cause the curled polymer chains to uncurl i.e. they become more linear. The change of conformation will increase the interaction between the chains and thereby the mobility in the film. Another consequence of this is that PEDOT:PSS, which is soluble in water, become (more) insoluble when ethylene glycol is added [12]. Another way to increase the conductivity is to add $\mathrm{ZnO}$ nanoparticles to PEDOT:PSS which has been reported to cause at least a doubling of the conductivity [17]. One of the first theoretical studies of the effect of doping on PEDOT:PSS was made by Dkhissi et al [18].

Another interesting application is the use of PEDOT:PSS in high broadband reflectivity materials such as low-emissive camouflage paints [19].

In theoretical studies of PEDOT and PEDOT:PSS in the literature [18,20-23] oligomers have been used to investigate the geometrical packing of PEDOT and its counter ions, as well as their interactions. Combined HF/6-31-G*, MP2/6-31++G** 
and forcefield calculations of a charged PEDOT octamer and a toluensulphonic acid counter ion showed that the perpendicular position of the counter ion to the PEDOT chain is the most stable of the studied positions [21]. Force field calculations indicate that the PEDOT and PSS polymers run parallel to each other [22].

In this paper we investigate the influence of the degree of doping on the reflectivity and other optical properties in PEDOT:PSS by employing quantum-chemical density functional theory (DFT) calculations. Such calculations can contribute to the understanding of some of the experimentally observed properties of PEDOT:PSS.

\section{Method}

Quantum-chemical electronic structure calculations and geometry optimizations of the structures and the cell sizes were performed by using the periodic density functional plane wave code CASTEP [24] in Materials Studio [25] with the GGA PW91 functional [26]. All geometry optimizations were performed using spin-polarized calculations where a $260 \mathrm{eV}$ plane-wave energy cut off was chosen. Brillouin zone integrations were performed using a tight k-point separation of $0.04 \AA^{-1}$.

A periodic PEDOT structure was built guided by the distances given for PEDOT doped with tosylate [27] and with $\mathrm{PF}_{6}$ [28]. The geometry optimized structure (cell axes and coordinates are optimized) of the PEDOT polymer is shown in Fig. 1a. For the poly(styrenesulfonate) polymer (in this work noted PSSH in its protonated neutral form and $\mathrm{PSS}^{-}$in its negatively charged state), Fig. 1b, the cell axes are optimized from a start guess were they were chosen to fit the optimized PEDOT cell. Theoretical calculations [22] for PEDOT:PSS oligomers indicate that the PSS polymer lies parallel to the PEDOT polymer. Here, we investigate two periodic structures which fulfill this condition, either with the PSSH polymer located between stacks of PEDOT polymers, Fig. 1c, or with PSSH forming separating layers between the PEDOT polymers, Fig. 2a. The two polymers are combined into one periodic cell with a start guess in which the distance between the PEDOT and the PSSH polymers was about one hydrogen bond length. Coordinates and cell axes are optimized for the two periodic PEDOT:PSSH structures. The structure with alternating PEDOT and PSSH polymers is chosen for the remaining study of electronic properties since this structure is found to be lower in energy $\left(-14.3 \mathrm{~kJ} \mathrm{~mol}^{-1}\right)$ compared to the structure with the 
PSSH between the PEDOT stacks $\left(-12.0 \mathrm{~kJ} \mathrm{~mol}^{-1}\right)$. By removing hydrogens from the PSSH polymer, but still keeping the total system neutral, the PSS polymer becomes negatively charged, i.e. is in the PSS $^{-}$form, and positive holes are introduced in the PEDOT polymer. From the Mulliken charges it is seen that the positive charge is mainly distributed at the carbon atoms in the conjugated chain in the PEDOT polymer, whose charges have been increased by about 0.05 , but also at the $\mathrm{O}$ and $\mathrm{S}$ atoms in both PEDOT (0.03 and 0.07) and PSS (0.02 and 0.08). The removal of the hydrogens is performed in two ways, either the hydrogens are removed at every second $\mathrm{SO}_{3}$ group at the PSSH polymer, Fig. 2b (this structure is referred to as $\mathrm{PEDOT}^{+0.5}: \mathrm{PSS}^{-} / \mathrm{PSSH}$ ), or all hydrogens are removed, Fig. 2c (referred to as $\mathrm{PEDOT}^{+}: \mathrm{PSS}^{-}$). This gives three investigated forms corresponding to successively increased doping: PEDOT:PSSH, PEDOT ${ }^{+0.5}: \mathrm{PSS}^{-} / \mathrm{PSSH}$, and $\mathrm{PEDOT}^{+}: \mathrm{PSS}^{-}$.

In terms of the electronic properties, it can be noted that the imaginary part of the dielectric function can be calculated, and then the real part can be derived by the Kramers-Kronig relationship. Using the complex dielectric function optical properties such as the reflectivity and absorbance can be calculated [29].

\section{Results and Discussion}

The optimized structures for the PEDOT and PSSH polymers are shown in Fig. 1. The structure for PEDOT:PSS with alternating PEDOT and PSS polymers is shown in Fig. 2 for both the structure case were both polymers are neutrally charged and the two structures with the positively doped PEDOT and the negatively charged PSS ${ }^{-}$ polymers acting as counter ions. Cell axes and densities are given in table 1 . The optimized cell axis are the same for PEDOT:PSSH and the two doped structures. The repeat unit of the PEDOT chain is calculated to be 7.7-7.8 $\AA$, and the lattice parameter which determines the distance between the chains stacked on top of each other is found to be $6.9 \AA$, Both these values agree with previously reported experimental results [27-28]. The separation between the PEDOT chains increases from $3.56 \AA$ in the pure PEDOT structure, to $3.83 \AA$ in PEDOT:PSSH, and to $3.82 \AA$ in the two

doped structures. This could be compared to the inter chain distance of $3.8 \AA$ obtained in ref. [30]. The calculated energy gain when the two polymers PEDOT and PSSH form the mixed PEDOT:PSSH structure is $-14.3 \mathrm{~kJ} \mathrm{~mol}^{-1}$ per PEDOT:PSSH unit. 
Selected distances and angles defined in Fig. 3 are given in table 2. The changes in bond lengths and angles are consistent with the transformation upon doping from a benzoid to the quinoid structure of PEDOT in accordance to the findings of Dkhissi et.al [20] and Ouyanga et. al [12]. In particular, the bond distance $a$, which in the benzoid structure corresponds to a double bond, is shorter in the undoped structure, whereas the bonds $b$ and $c$, corresponding to double bonds in the quinoid structure, are shortened in the doped structures. Lapowski and Pron [31] reported from Raman measurements the quinoid structure for the undoped state. Their result contradicts the present result and was also questioned by Dkhissi et.al [20].

Electronic densities of states (DOS) graphs for PEDOT:PSSH, PEDOT $^{0.5+}: \mathrm{PSS}^{-} / \mathrm{PSSH}$ and PEDOT ${ }^{+}$PSS $^{-}$are shown in Fig. 4 where projection on the atoms of the PEDOT polymer is marked by the filled area. The Fermi level of PEDOT:PSSH is set to $0 \mathrm{eV}$

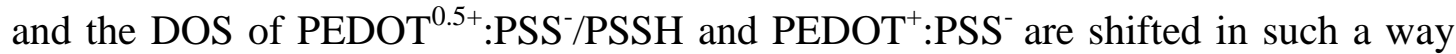
that the orbitals with peaks at -1.6-2.1 eV for the undoped polymers are situated at the same energy for the doped polymers. The orbitals corresponding to HOMO and LUMO in the three levels of doping are shown in Fig. 5 together with the lowest and highest energy for each band. In the DOS in Fig. 4 each of these bands turn up as two seemingly separate peaks at the energies of the band's low- and high energy endpoints. In the undoped case the HOMO band is located at PEDOT. The lowenergy endpoint of the LUMO band is located mainly on PEDOT, whereas the highenergy endpoint is located at PSSH (see Figs. 4 and 5). For the doped structures some bands are partially filled. The formal HOMO and LUMO bands are both partially filled for $\mathrm{PEDOT}^{0.5+}: \mathrm{PSS}^{-} / \mathrm{PSSH}$. For $\mathrm{PEDOT}^{+}$PSS $^{-}$the formal HOMO is slightly less than doubly occupied and as a consequence the LUMO and LUMO+1 are also partially occupied. The occupied parts of the partially filled bands are localized mainly at the PSS polymer, whereas the unoccupied part is localized mostly at the PEDOT. DFT calculations generally underestimate the band gap, which is also the case here where the calculated band gap for the undoped structure is $0.7 \mathrm{eV}$ compared to the experimental band gap [1,32] of $1.5 \mathrm{eV}$. This can be compared to the band gap in ref. [18] of $1.65 \mathrm{eV}$ for an infinite polymer that was obtained by extrapolation of the first excitation energy for 2-10 oligomers from semiempirical INDO with singles configurations calculations. From the B3LYP calculations of the HOMO-LUMO gap for oligomers of lengths up to 8 units in ref [23] one can deduce a band gap at infinite 
length of about $2.2 \mathrm{eV}$, which probably is an overestimation that can be attributed to the incorporation of part of the exact electron exchange interactions in that hybrid functional. We note from refs $[18,23]$ that using an 8-oligomer as a model for the polymer of infinite length gave an overestimation of the band bap of about $0.4-0.5 \mathrm{eV}$. Using the method in the present study the calculated band gap for the 8-oligomer is $1.3 \mathrm{eV}$, i.e. about $0.6 \mathrm{eV}$ larger than that of the infinite polymer.

The calculated reflectivities for the PEDOT:PSS at the three studied degrees of doping are shown in Fig. 6. At higher energies/shorter wavelengths the reflectivity is not affected by the introduced doping. At long wavelengths/low energies the doping of the PEDOT polymer influences the reflectivity significantly. For the uncharged PEDOT:PSSH polymers the calculated reflectivity is relatively low, only about 0.05 . This increases to 0.3 when PEDOT is doped with one positive charge at each second EDOT monomer. Increasing the doping to one positive charge at each monomer causes the reflectivity to increase significantly to about 0.7 . Molecular vibrations which are not included here would contribute to the fine structure of the spectra at low energies/high wavelengths, below $0.5 \mathrm{eV} /$ above $2.5 \mu \mathrm{m}$. Measurements of the reflectivity for PEDOT:PSS coated PET foils [33] show that there are peaks at $1.4 \mathrm{eV}$, $2.5 \mathrm{eV}$ and $3.6 \mathrm{eV}$ and a minimum corresponding to $1.0 \mathrm{eV}$. A similar structural behaviour can be seen in the calculated reflectivity in Fig. 6a where peaks for the undoped structure are seen at $1.5 \mathrm{eV}, 2.7 \mathrm{eV}$ and $3.3 \mathrm{eV}$ and at $2.5 \mathrm{eV}$ and $3.1 \mathrm{eV}$ for the doped structure. The minimum in the experimental spectrum could possibly be caused by the existence of a mixture of the doped and undoped structures, since the sum of the calculated spectra for the PEDOD:PSSH and the PEDOT ${ }^{+}: \mathrm{PSS}^{-}$structures display a such minimum (Fig. 6).

Experimental studies [32] of PEDOT with $\mathrm{PF}_{6}$ counter ion show that the reflectivity decreases from about 0.8 at low energies to 0.1 at $4 \mathrm{eV}$ with a minimum at $2 \mathrm{eV}$, which approximately agrees with the calculated results. When the polarized reflectivity is studied, Fig. 7, it is seen that the main part of the reflectivity stems from

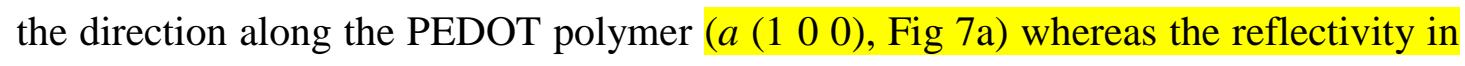
the other directions is much smaller (Fig 7b). This can also be inferred from the energy band structure shown in Fig. 8 where the dispersion along the $a$ (B-G) direction which is parallel to the PEDOT chain, is much larger than in the orthogonal 
directions. As a result it will be the bands in the $a$ direction that become partially filled, i.e. conducting in character.

In Fig. 9 the calculated absorbance, real and imaginary parts of the refractive index, dielectric function and conductivity and the energy-loss function are shown. Since the calculated optical properties are based only on the electronic energy calculations vibrational effects at low energies are missing in the calculated spectra. The calculated conductivity displays peaks at $0.16 \mathrm{eV}$ and $0.64 \mathrm{eV}$ for PEDOT $^{+}:$PSS $^{-}$and a peak at $1.3 \mathrm{eV}$ for PEDOT:PSSH. The optical conductivity for PEDOT:PF 6 obtained from experimentally measured reflectance [32] shows peaks at $0.1,0.4$ and $1.5 \mathrm{eV}$. The peaks in the calculated energy loss-function mirror the energies where the reflectivity vanishes.

\section{Conclusions}

A PEDOT:PSSH crystalline structure was modeled by periodic quantum-chemical calculations. The most stable structure is found to be a structure with alternating PEDOT and PSS polymers. A transformation from the benzoid to the quinoid structure is observed for the PEDOT chain when the neutral PEDOT is doped. Doping was seen to result in a removal of electrons from mainly the PEDOT $\pi$-conjugated system as expected. Doping was found to have significant effects on the electronic structure. The reflectivity was seen to increase considerably with increased doping, and also other optical spectral properties were appreciable affected.

\section{Acknowledgements}

The Swedish Armed Forces is acknowledged for financing a part of the project. Financial support from the Swedish Research Council, VR, and computational resources from the Swedish National Supercomputer Centre, NSC, is acknowledged. 


\section{References}

[1] Q. Pei, G. Zuccarello, M. Ahlskog, O. Inganäs, Polymer 35 (1994) 1347.

[2] S. Kirchmeyer, K. Reuter, J. Mater. Chem. 15 (2005) 2077.

[3] L. Bert Groenendaal, F. Jonas, D. Freitag, H. Pielartzik, J.R. Reynolds, Adv. Mat. $12(2000) 481$.

[4] L. Groenendaal, G. Zotti, F. Jonas, Synth. Met. 118(2001) 105.

[5] C.K. Chiang, C.R. Fischer, Y.W. Park, A.J. Heeger, H. Shirakawa, E.J. Louis,

S.C. Gau and A.G. MacDiarmid, Phys. Rev. Letters 39 (1977) 1098

[6] N. Koch, A. Vollmer, A. Elschner, Appl. Phys. Lett. 90 (2007) 043512.

[7] M.H. Bolin, K. Svennersten, X. Wanga, I.S. Chronakis, A. Richter-Dahlfors, E.W.H Jager, M. Berggren, Sens. Actuators, B 142 (2009) 451.

[8] J. Hupe, G.D. Wolf, F. Jonas, Galvanotechnik 86 (1995) 3404.

[9] A. Laforgue, J. Mater. Chem. 20 (2010) 8233.

[10] N. Koch, A. Kahn, J. Ghijsen, J.-J. Pireaux, J. Schwartz, R.L. Johnson, A. Elschner, Appl. Phys. Lett. 82 (2003) 70.

[11] M. Halik, H. Klauk, U. Zschieschang, G. Schmid, W. Radlik, S. Ponomarenko, S. Kirschmeyer, J. Appl. Phys. 93 (2003) 2977.

[12] J. Ouyanga, Q. Xua, C.-W. Chua, Y. Yanga, G. Lib, J. Shinar, Polymer 45 (2004) 8443.

[13] J.Y. Kim, J.H. Jung, D.E. Lee, J. Joo, Synth. Met. 125 (2002) 311.

[14] L.A.A. Pettersson, S. Ghosh, O. Inganäs, Org. Electron. 3 (2002) 143.

[15] S.K.M. Jönsson, J. Birgersson, X. Crispin, G. Grecynski, W. Osikowicz, A.W.D. van der Gon, W.R. Salaneck, M. Fahlman, Synth. Met.139 (2003) 1. 
[16] X. Crispin, S. Marciniak, W. Osikowicz, G. Zotti, A.W. D. van der Gon, F. Louwet, M. Fahlman, L. Groenendaal, F. de Schryver, W.R. Salaneck, J. Polym. Sci., Polym. Phys. 41 (2003) 2561.

[17] N.G. Semaltianos, S. Logothetidis, N. Hastas, W. Perrie, S. Romani, R.J. Potter, G. Dearden, K.G. Watkins, P. French, M. Sharp, Chem. Phys. Lett. 484 (2010) 283.

[18] A. Dkhissi, D. Beljonne, R. Lazzaroni, F. Louwet, L. Groenendaal, J. L. Brédas, Int. J. Quantum. Chem. 91 (2003) 517

[19] E. Hedborg Karlsson, H. Kariis, Å. Andersson, C. Åkerlind, FOI-rapport, FOI-R-2326--SE, Augusti 2007.

[20] A. Dkhissi, F. Louwet, L. Groenendaal, D. Beljonne, R. Lazzaroni, J. L. Brédas, Chem. Phys. Lett. 359 (2002) 466

[21] A. Dkhissi, D. Beljonne, R. Lazzaroni, F. Louwet, B. Groenendaal, Theor. Chem. Account. 119 (2008) 305.

[22] A. Dkhissi, D. Beljonne, R. Lazzaroni, Synth. Met.159 (2009) 546.

[23] C. Alemán, E. Armelin, J.I. Iribarren, F. Liesa, M. Laso, J. Casanovas, Synth. Met. 149 (2005) 151.

[24] D. Vanderbilt, Phys. Rev. B 41 (1990) 7892.

[25] Accelrys MS Modeling Getting Started, manual Release 4.0, Accelrys Software Inc.: San Diego, 2006.

[26] J.P. Perdew, J.A. Chevary, S.H. Vosko, K.A. Jackson, M.R. Pederson, D.J. Singh, C. Fiolhais, Phys. Rev. B 46 (1992) 6671.

[27] K.E. Aasmundtveit, E.J. Samuelsen, L.A.A. Pettersson, O. Inganäs, T. Johansson, R. Feidenhans'1, Synth. Met. 101 (1999) 561.

[28] L. Niu, C. Kvarnström, K. Fröberg, A. Ivaska, Synth. Met. 122 (2001) 425.

[29] S. Saha, T.P. Sinha, A. Mookerjee, Phys. Rev. B 62 (2000) 8828. 
[30] A. Dkhissi, D. Beljonne, R. Lazzaroni, F. Louwet, B. Groenendaal, Theor Chem Account 119 (2008) 305

[31] M. Lapowski, A. Proń, Synth. Met. 110 (2011) 79

[32] Y. Chang, K. Lee, R. Kiebooms, A. Aleshin, A.J. Heeger, Synth. Met. 105 (1999) 203.

[33] Jürgen Jung, "Optical properties of PEDOT:PSS coatings", Agfa internal report (2009), Agfa Gevaert NV / Belgium, www.agfa.com 
Table 1

Cell sizes and densities for the geometry-optimized structures.

\begin{tabular}{lcccc}
\hline Cell & $a(\AA)$ & $b(\AA)$ & $c(\AA)$ & Density $\left(\mathrm{g} \mathrm{cm}^{-3}\right)$ \\
\hline HPSS & 7.7 & 6.9 & 16.9 & 1.36 \\
PEDOT & 7.8 & 11.8 & 6.9 & 1.47 \\
PEDOT:PSSH & 7.7 & 11.7 & 22.5 & 1.06 \\
PEDOT $^{0.5+}$ :PSS $/$ PSSH & 7.7 & 11.7 & 22.5 & 1.06 \\
PEDOT $^{+}:$PSS $^{-}$ & 7.7 & 11.7 & 22.5 & 1.06 \\
\hline
\end{tabular}




\section{Table 2}

Average bond lengths and angles in the PEDOT polymer for the three PEDOT:PSS structures. a-f as given in Fig. 3, $\mathrm{g}$ is the torsion angle between the PEDOT monomers.

\begin{tabular}{cccc}
\hline & PEDOT:PSSH & PEDOT $^{+0.5}:$ PSS $^{-} /$PSSH & PEDOT $^{+}:$PSS $^{-}$ \\
\hline $\mathrm{a}$ & $1.38 \AA$ & $1.40 \AA$ & $1.40 \AA$ \\
$\mathrm{b}$ & $1.40 \AA$ & $1.38 \AA$ & $1.38 \AA$ \\
$\mathrm{c}$ & $1.41 \AA$ & $1.39 \AA$ & $1.39 \AA$ \\
$\mathrm{d}$ & $128.7^{\circ}$ & $127.2^{\circ}$ & $126.8^{\circ}$ \\
$\mathrm{e}$ & $113.5^{\circ}$ & $113.6^{\circ}$ & $113.5^{\circ}$ \\
$\mathrm{f}$ & $92.7^{\circ}$ & $92.3^{\circ}$ & $92.2^{\circ}$ \\
$\mathrm{g}$ & $169.6^{\circ}$ & $171.3^{\circ}$ & $171.3^{\circ}$ \\
\hline
\end{tabular}


Fig. 1. Optimized structures for a) PEDOT, b) PSSH and c) PEDOT:PSSH with PSSH between the stacks of PEDOT polymers. (Cell axes, PEDOT:PSSH, 7.8x6.9x33.9 $\AA$, density $1.18 \mathrm{~g} \mathrm{~cm}^{-3}$ )

Fig. 2. Optimized structures for a) PEDOT:PSSH, b) $\mathrm{PEDOT}^{0.5+}: \mathrm{PSS}^{-} / \mathrm{PSSH}$ and c) $\mathrm{PEDOT}^{+}: \mathrm{PSS}^{-}$.

Fig. 3. Labels of the bond lengths and angles whose numerical values are given in Table 2. The atom charge increments due to the doping are also given.

Fig. 4. DOS for PEDOT:PSSH, $\mathrm{PEDOT}^{0.5+}: \mathrm{PSS}^{-} / \mathrm{PSSH}$ and $\mathrm{PEDOT}^{+}: \mathrm{PSS}^{-}$where the filled area corresponds to PDOS for the PEDOT polymer. The dotted line indicates the Fermi level.

Fig. 5. Molecular orbitals for PEDOT:PSSH, PEDOT ${ }^{0.5+}: \mathrm{PSS}^{-} / \mathrm{PSSH}$ and PEDOT $^{+}:$PSS $^{-}$. The orbitals to the left of the bold line are occupied. For each orbital the lowest/highest value of the energy of the band at any k-point is given in $\mathrm{eV}$.

Fig. 6. Calculated reflectivity a) as a function of energy (eV) and b) as a function of wavelength (nm)

Fig. 7. Calculated polarized reflectivity as a function of energy (eV) for

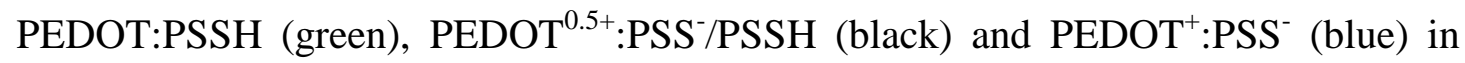
the $a\left(\begin{array}{lll}1 & 0 & 0\end{array}\right)$ and $b\left(\begin{array}{lll}0 & 1 & 0\end{array}\right)$ directions.

Fig. 8. The calculated energy band structure of a) PEDOT:PSSH and b)

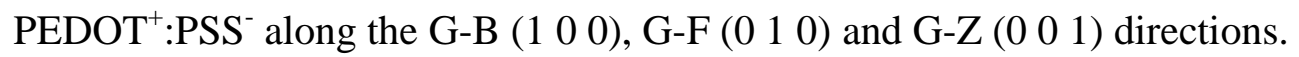

Fig. 9. Calculated optical parameters of PEDOT:PSSH (green), $\mathrm{PEDOT}^{0.5+}{ }^{\mathrm{PSS}^{-}}$ /PSSH (black) and PEDOT $^{+}:$PSS $^{-}$(blue). Absorbance I, real part of the refractive index $n$, imaginary part of the refractive index $k$, the real and imaginary part of the dielectric function $\varepsilon_{1}$ and $\varepsilon_{2}$, the real and imaginary part of the conductivity $\sigma_{1}$ and $\sigma_{1}$ and the energy-loss function $L$. 
a)

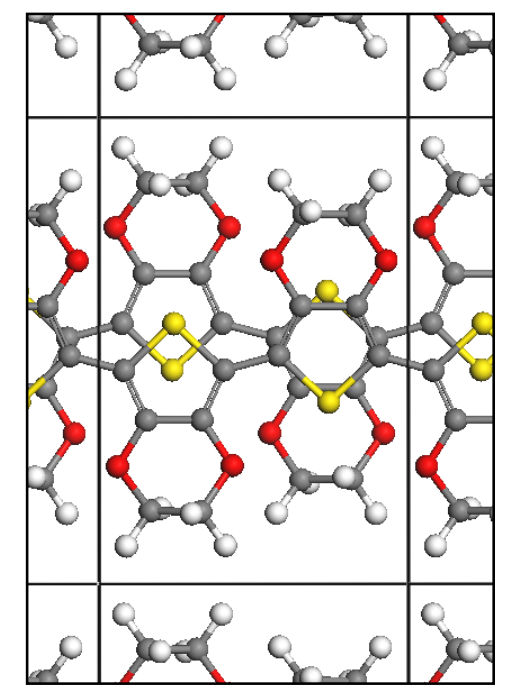

b)

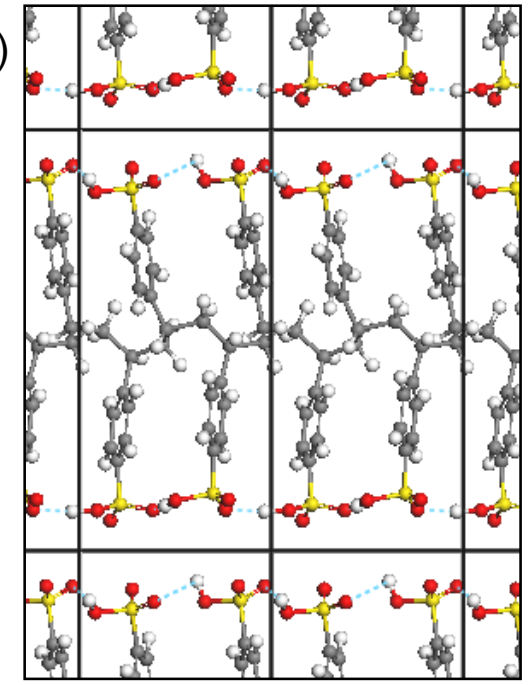

c)
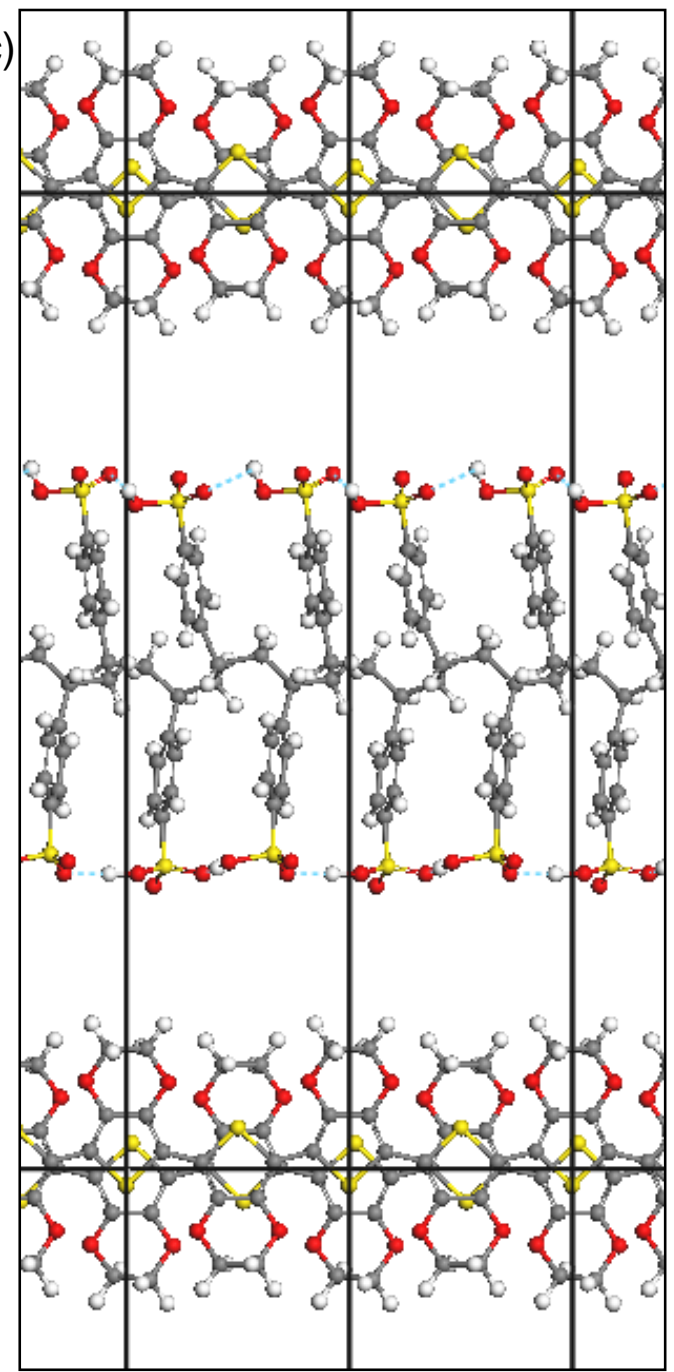

Fig. 1. 
a)

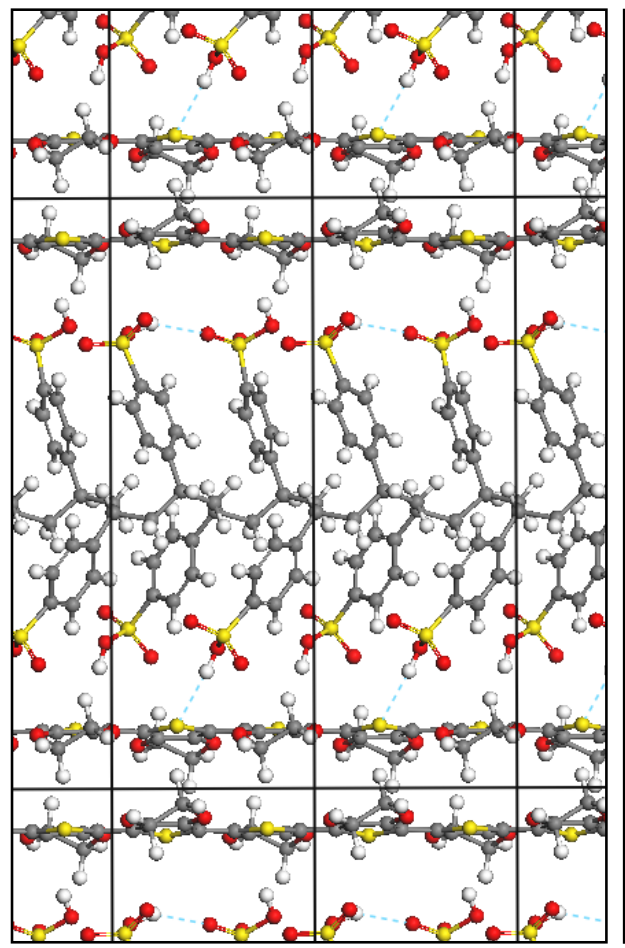

b)

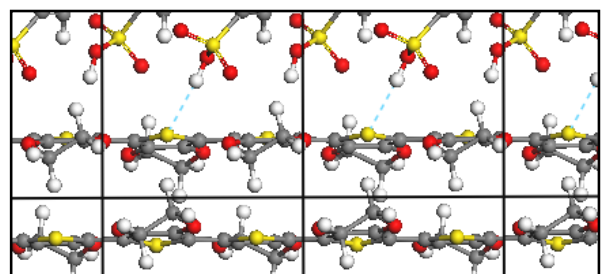

c)

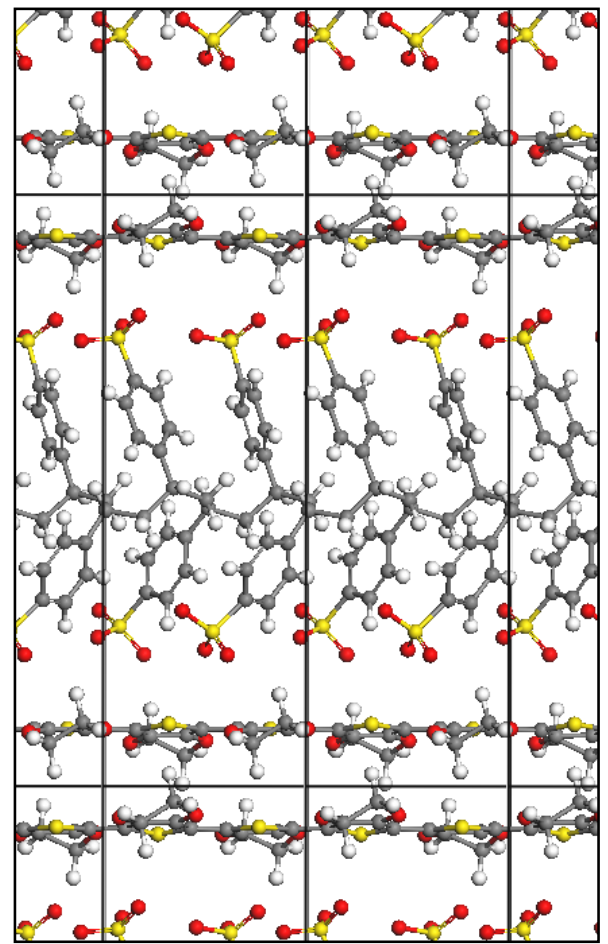

Fig. 2. 


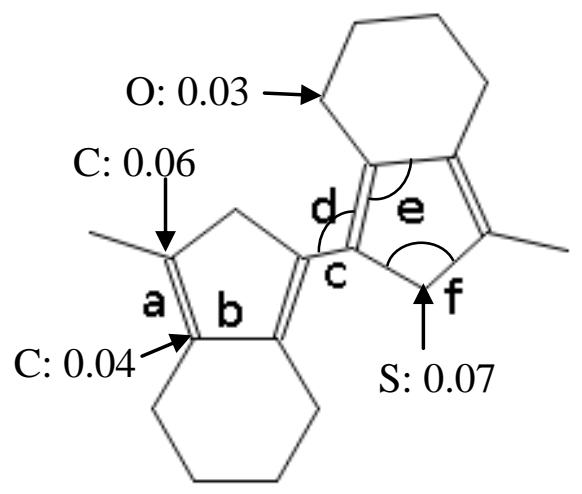

Fig. 3. 


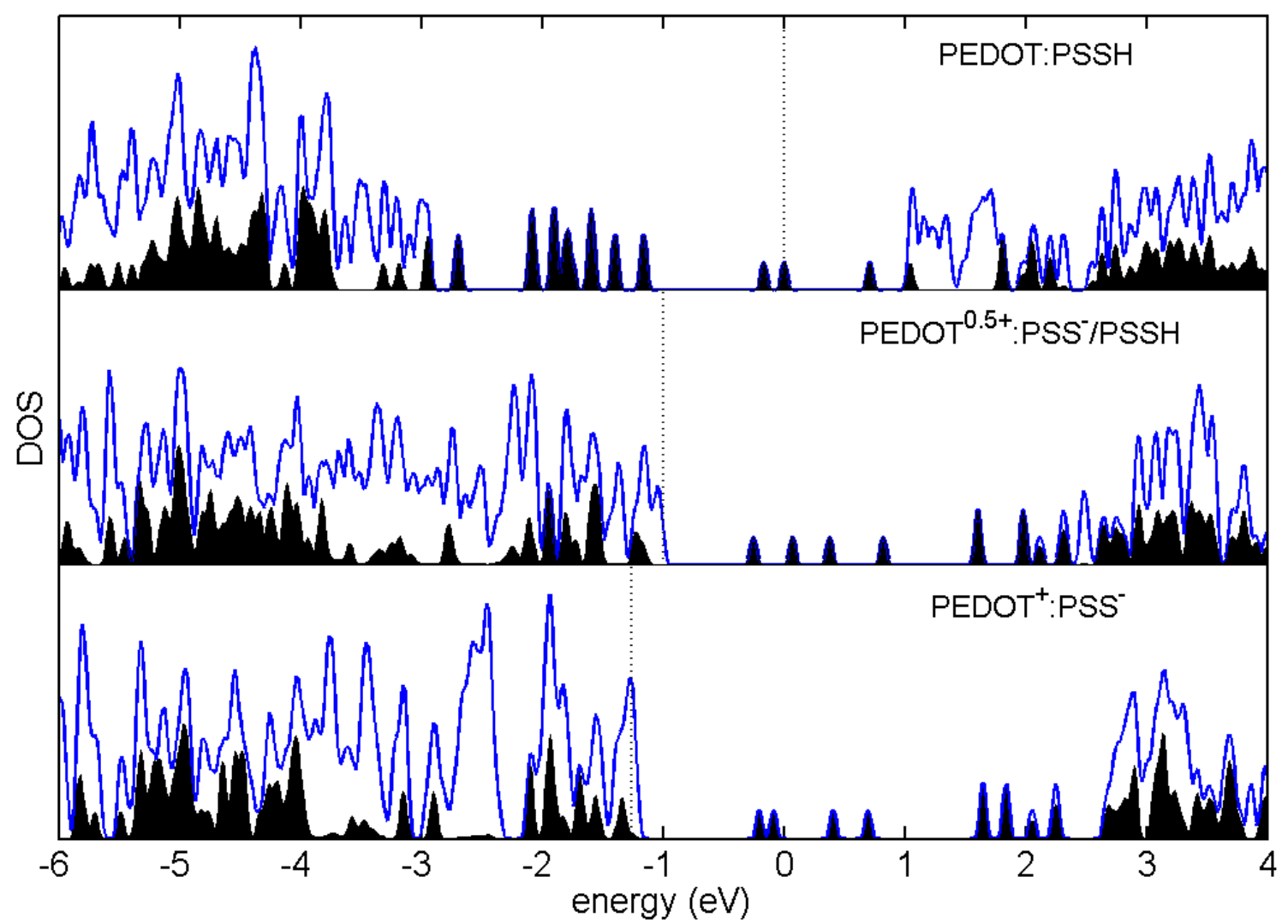

Fig. 4. 


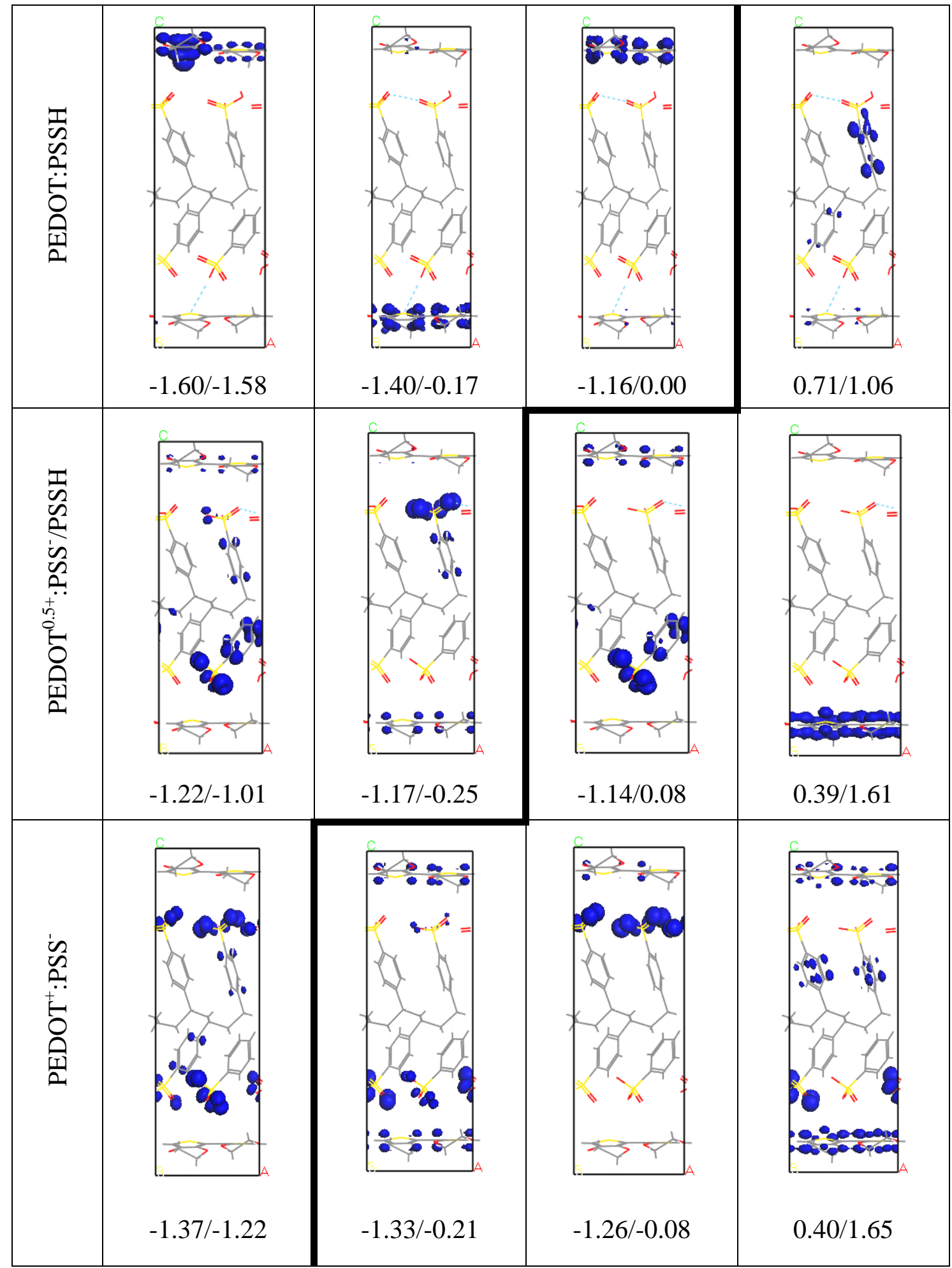

Fig. 5. 

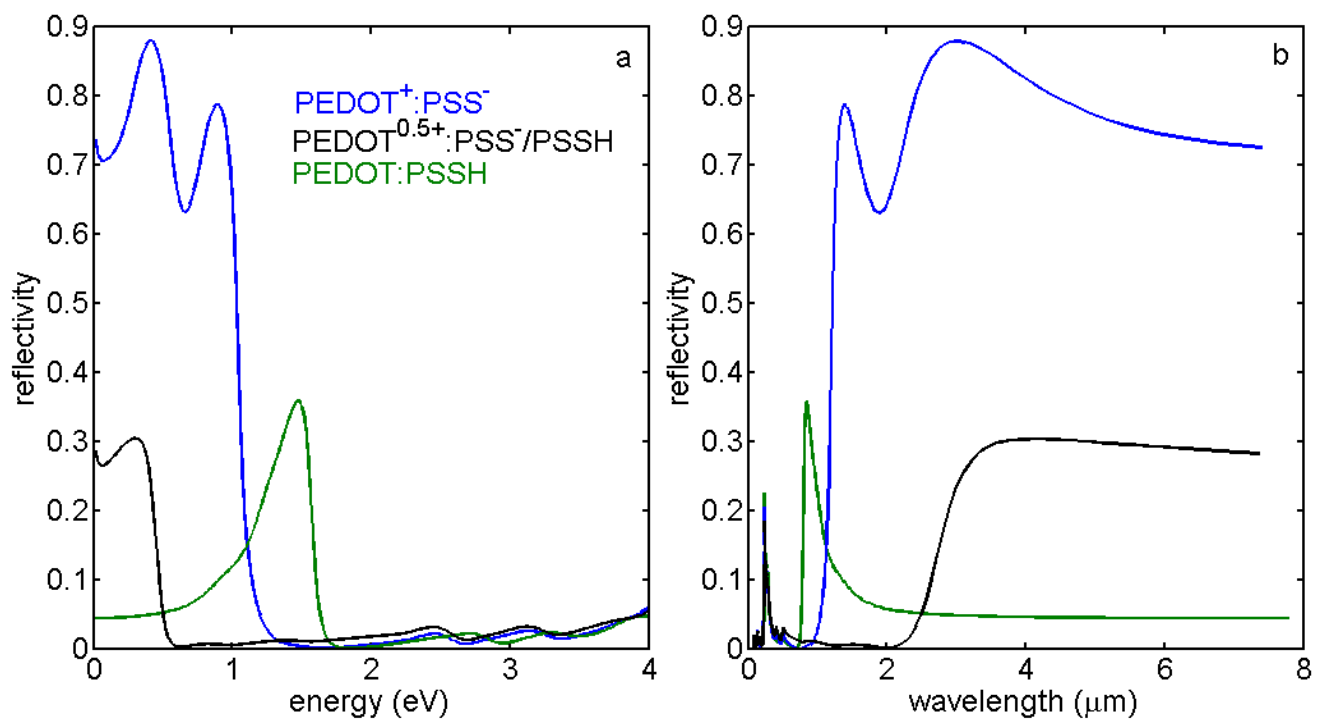

Fig. 6. 


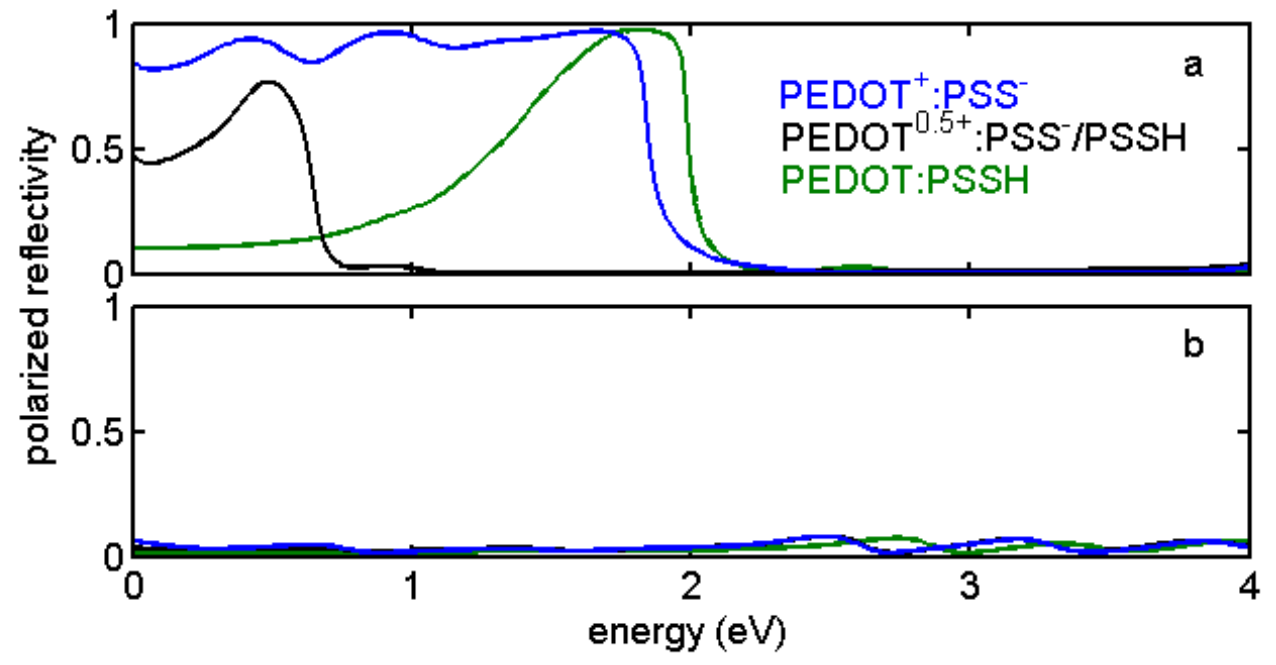

Fig. 7. 

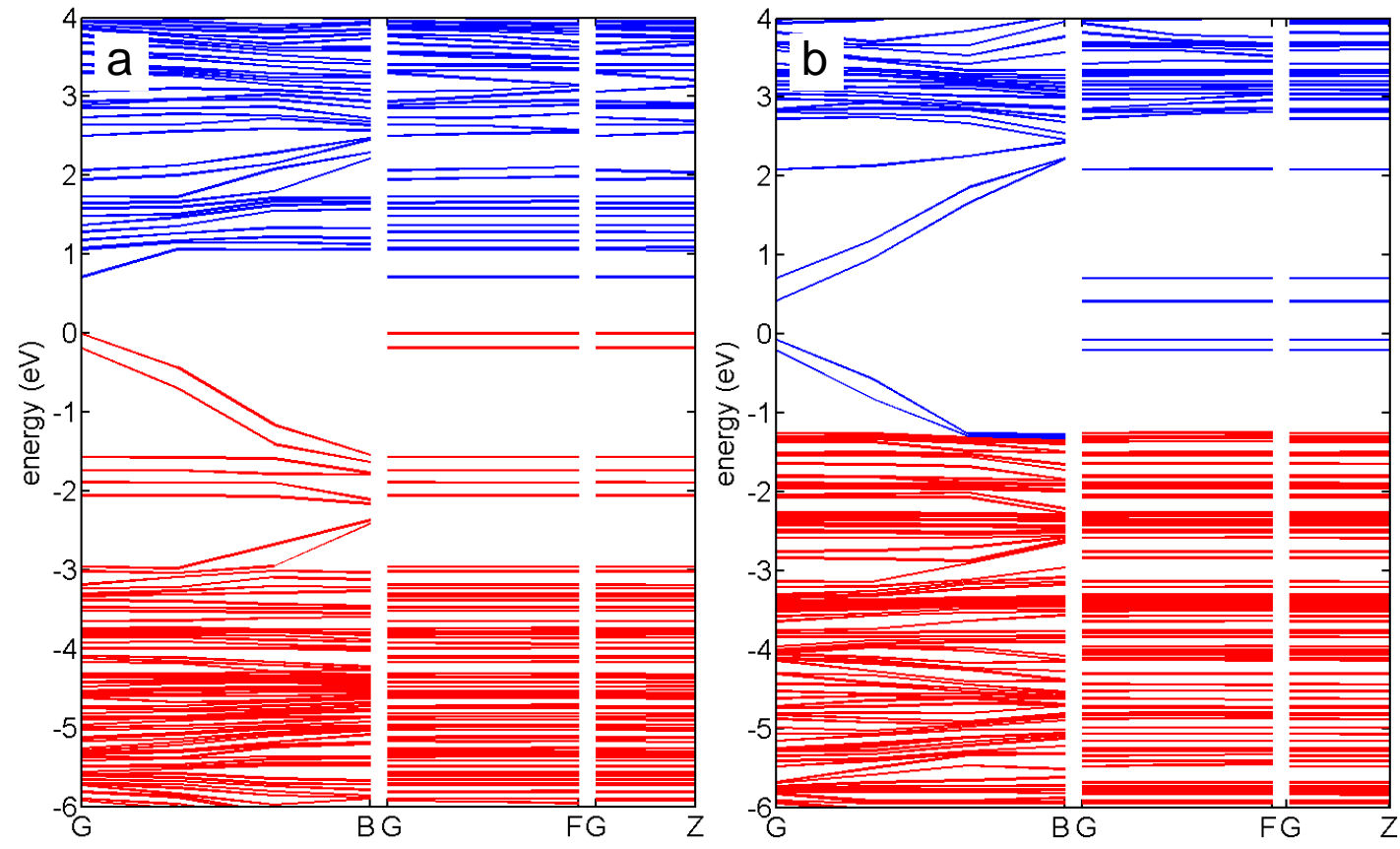

Fig. 8. 

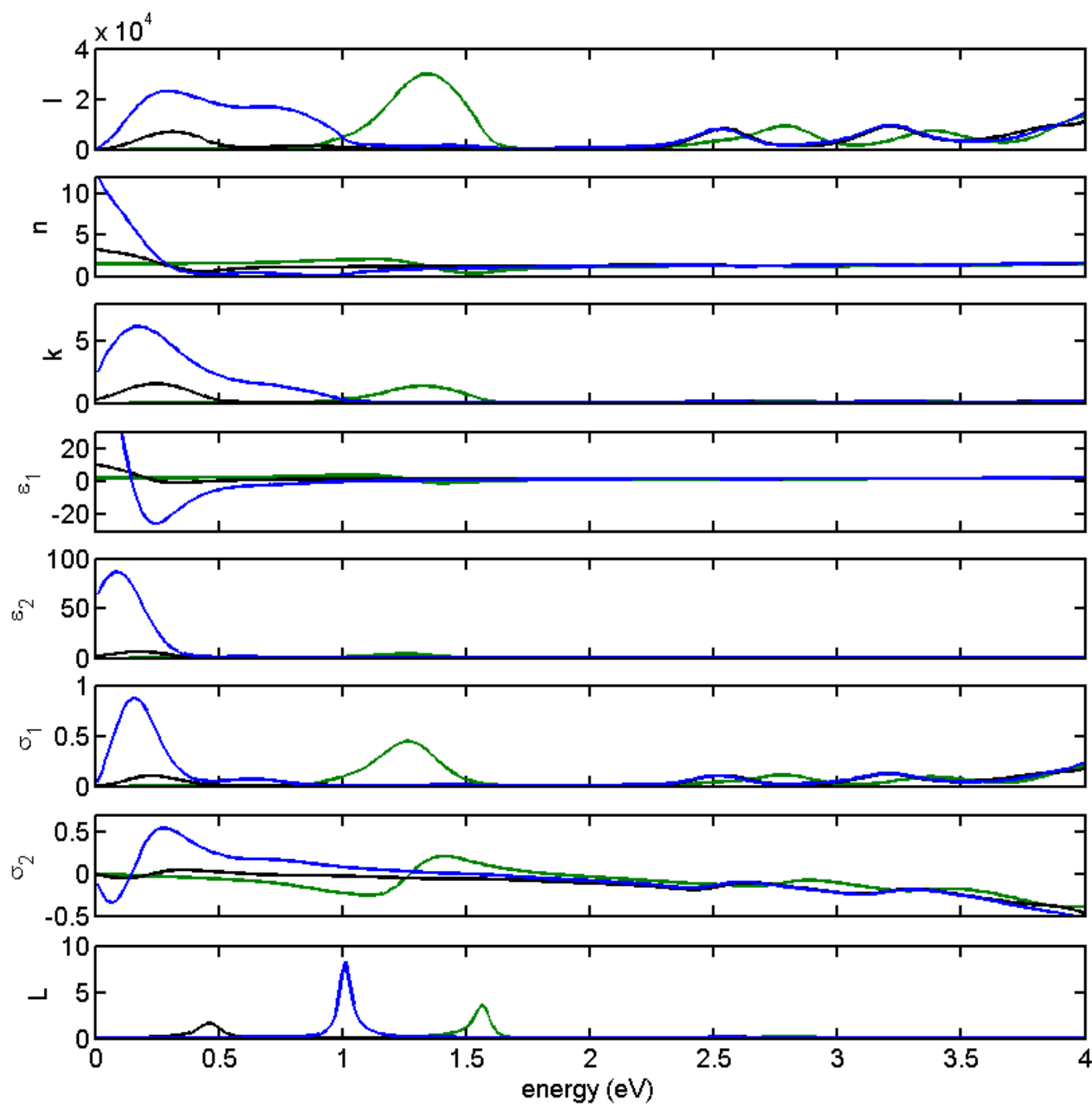

Fig. 9. 